Military Technical College

Kobry El-Kobba

Cairo, Egypt

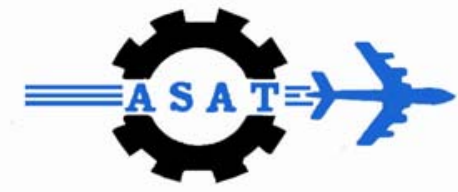

12-th International Conference

on

Aerospace Sciences \&

Aviation Technology

\title{
DEVELOPMENT AND TESTING OF AN INNOVATIVE MINI-UAV CONFIGURATION
}

\author{
GUGLIERI* G. and QUAGLIOTTI* F.
}

\begin{abstract}
The MicroHawk research program is outlined in the present paper. The evolution of the research program, the design phases, the theoretical and the experimental work are detailed. The research and development activities are presented including the production of the industrial versions of the platform.
\end{abstract}

\section{KEY WORDS}

Unmanned Aerial Vehicles, Aircraft Design, Flight Testing

\section{INTRODUCTION}

In the last decade the term MAVs (Micro Aerial Vehicles) has been used to define flying objects characterized by reduced physical sizes and can be thought of as aerial robots, as six-degree-of-freedom machines whose mobility can deploy a useful micro payload to a remote or otherwise hazardous location where it may perform any of a variety of missions [1]. The geometric dimension constraint puts MAVs at least an order of magnitude smaller than any UAV platform developed to date, as Fig. 1 shows. Therefore, due to the reduced dimensions, MAVs can be also intended as payload of greater uninhabited aerial vehicles, with the important characteristics of autonomous flight and mission independence. MAVs project and their applications are topical subjects, involving design and technology challenges, which several research centers/universities and industries are currently facing with (see [2] and [3] for an overview).

MAVs will be capable of a wide range of useful missions, both in military and civilian fields. Because of their size, the aircraft will be able to perform unconventional missions not compatible with any existing platform. Among military applications: "over the hill" reconnaissance, surveillance, targeting, communications and weapons-like functionality. Several civilian missions can be numbered: localization of missing people in wild areas, reconnaissance and surveillance of territory such as steep slopes and narrow places, traffic monitoring, fire and rescue operations, biochemical sensing and sensor placement.

* Politecnico di Torino/DIASP-MAVTech s.r.I., Torino, Italy. 


\section{RESEARCH ACTIVITY}

The MicroHawk concept was designed within a European Union funded project (MARVEL Micro Aerial Vehicles for Multi Purpose Remote Monitoring and Sensing Project), by a research group at Politecnico di Torino. The MicroHawk configuration is characterized by a conventional layout: it is a fixed wing, tailless integrated wing-body configuration and tractor propeller driven.

Fixed wing configurations have known so far a wider application because of their larger payload capabilities, the flight performances, such as mission range and endurance, and their capability to better withstand to adverse weather conditions with respect to the other existing micro and mini air vehicle configurations.

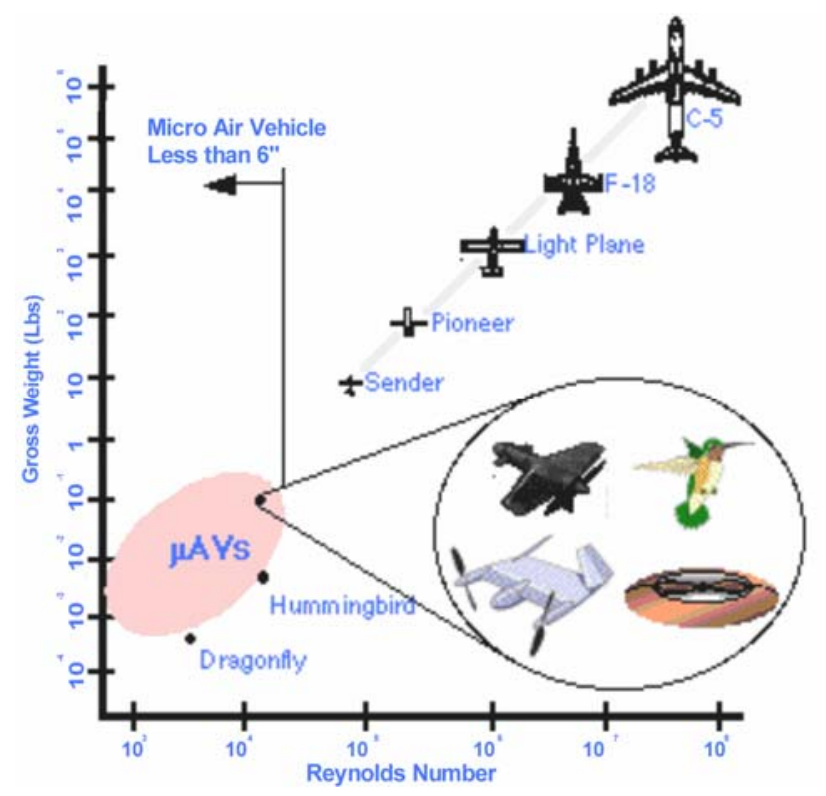

Fig. 1. Flight regimes characteristics of different classes of air vehicles.

The allwing configuration has several advantages: the design is compact with an adequate aerodynamic efficiency, masses and subsystems are concentrated, structures are light and severe aeroelastic problems are rejected as no tailplane is present, stall is smooth and the configuration is spin resistant and stable in flight. Some disadvantages: high lift devices are difficult to apply and the platform has a limited acceptable range for payload location due to stability margin constraints.

Different versions of the MicroHawk configuration exist, covering a range of dimensions and operating performances. The main geometric dimensions of the MicroHawk versions are summarized in Tab. 1. The availability of different MicroHawk versions allows covering an extended range of flight speed (estimated between $7 \mathrm{~m} / \mathrm{s}$ and 20 $\mathrm{m} / \mathrm{s}$ ) and flight endurance (ranging between 15 and $60 \mathrm{~min}$, according to size and energy source storage). Performances are summarized in Tab. 2. 
A $150 \mathrm{~mm}$ wingspan platform - named $\mathrm{MH} 150$ - has been designed and developed for very short-range, remotely piloted missions, characterized by low flight duration and narrow operating scenario. It has been equipped with basic on-board systems (DC motor, propeller, battery pack, controller, receiver and servos), for a total weight of approximately 35 grams.

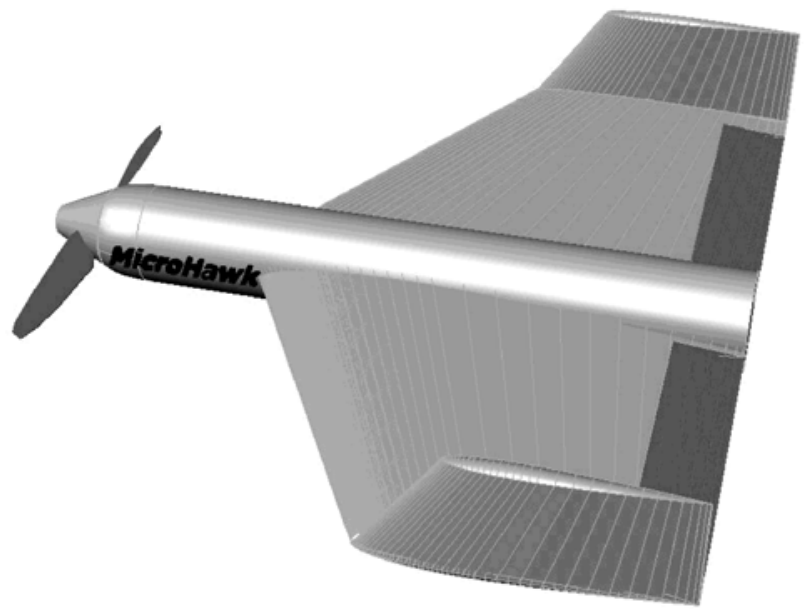

Fig. 2. MicroHawk design.

A larger platform - named MH300 - is characterized by a $337 \mathrm{~mm}$ wingspan; it weights about 100 grams in the unequipped version. The MH300 has been developed to perform a basic reconnaissance mission, carrying a micro camera and/or sensors for remote monitoring and sensing.

The medium size vehicle - named MH600 - is characterized by a $600 \mathrm{~mm}$ wingspan. Its design has been mainly addressed to the need for higher payload weight fraction and larger internal volumes. The MH600 version can achieve autonomous flight, as it is possible to locate on-board a commercial small size autopilot (Micropilot MP2028) without exceeding wing loading limitations for hand launch. The navigation system, based on inertial and GPS data, is programmable according to tasks and waypoints.

A larger platform (MH1000) was designed within a two-year research project (MURST/COFIN) for the development of a real time control and monitoring system for fire prevention. Autonomous programmable flight is a feature of this platform. On-board systems are expected to provide IR-imaging and visual streaming of territory. The vehicle is hand launched and after the take-off a datalink is established with the ground control station through a radio serial communication protocol for trajectory tracking and control. Advanced techniques for the design of flight control and navigation systems were also widely investigated.

A new research activity supported by PNRA (Programma Nazionale di Ricerche in Antartide) is expected to promote the application of innovative exploration techniques (such as autonomous flying vehicles) within the scientific activities performed by the 
Italian researchers in Antarctica. Within this area of interest a large platform (MH2000 with a $2000 \mathrm{~mm}$ wingspan) is under development. The basic concept is the aerial support to continental and maritime observation performed by means of an unmanned vehicle remotely controlled by an operator. Take-off and landing on a smooth surface are foreseen in remotely piloted or autonomous mode. The landing gear is equipped with wheels and skids for operation on snowy spots. An internal combustion engine is expected to provide the power for driving the tractor propeller. A communication link with a ground control station based on satellites is also considered. The payload is hosted within the fuselage in a modular section. The typical payload is a set of scientific instruments (i.e. magnetic field sensors) that will acquire data to be stored on-board during the flight. Heating systems for payload bay is under development. An alternative to conventional landing is also provided using a flight termination system that after engine cut off will deploy a decelerator (parachute). This option is also mandatory for failures and mission abort. Different $\mathrm{MH} 2000$ versions (powered by a DC motor) will be shortly available for surveillance, one of them being equipped with micro fuel cells.

\section{DESIGN PROCESS}

The design process of the vehicle started from aerodynamics, as the initial task was to relate $2 \mathrm{D}$ aerodynamics of aircraft wing section to aerodynamic characteristics of the aerial vehicle configuration and to evaluate its influences on flight performances. An analysis of the existing airfoil sections for low Reynolds number applications has been carried out in order to choose the suitable wing section and has been referred to experimental data. In fact, it is well known that the laminar separation bubble phenomena, characterizing the behavior of the low Reynolds number flows, affect the wing section performances and reduce the reliability of the most common methods for aerodynamic analysis. Wind tunnel flow visualizations on different wing sections were performed [4] in order to understand transitional and separation mechanisms for different flight conditions.

\begin{tabular}{|l|c|c|c|c|}
\hline & MH300 & MH600 & MH1000 & MH2000 \\
\hline WING & & & & \\
Wingspan [m] & 0.338 & 0.600 & 1.000 & 2.000 \\
Sweep [deg] & 30 & 30 & 30 & 30 \\
FUSELAGE & & & & \\
Length [m] & 0.29 & 0.52 & 0.875 & 1.750 \\
Width [m] & 0.03 & 0.055 & 0.075 & 0.143 \\
\hline
\end{tabular}

Table 1. MicroHawk layout geometry.

Because of the low aspect ratio characterizing the configuration under investigation, MicroHawk entails fully three-dimensional aerodynamics. The aerodynamic analysis of the 3D configuration [5] has been carried out by means of ACInterface ${ }^{\mathrm{TM}}$, a software code based on the extended lifting line theory (Weissinger theory), that can be used to evaluate the effects of any wing planform in incompressible flow. The software code has been used for the aerodynamics evaluation both of the wing and the vertical tail 
surfaces; it also performs a first approximation computation of the fuselage contribution to the aircraft aerodynamics according to the potential theory. ACInterface ${ }^{\mathrm{TM}}$ is a Java ${ }^{\mathrm{TM}}$ based software package [6] designed for the preliminary subsonic aerodynamic analysis of conventional and tailless aircraft. The data entry procedure allows to define the geometry, the propulsion system and the mass distribution of the configuration. As a result, the aerodynamic coefficients and the overall aircraft dataset are stored in formatted files either ready for graphics or compatible with Hexagon ${ }^{\mathrm{TM}}$ flight simulator.

\begin{tabular}{|l|c|c|c|c|c|}
\hline & MH300 & MH600 & MH1000 & MH2000 & MH2000 \\
\hline Autopilot & NO & NO/YES & YES & NO/YES & YES \\
Landing gear & NO & NO & NO & YES & YES \\
Propulsion & DC & DC & DC & DC & ICE \\
Speed range [m/s] & $7 \div 15$ & $7 \div 15$ & $7 \div 20$ & $7 \div 20$ & $7 \div 20$ \\
Empty weight [g] & 105 & $475 / 550$ & - & 6500 & 6500 \\
Payload weight [g] & 25 & $100 / 25$ & - & 2500 & 1500 \\
Altitude [m] & 100 & 100 & 100 & 100 & 100 \\
Typical range [km] & 0.3 & $0.3 / 2.5$ & $0.3 / 5$ & $0.3 / 7.5$ & 15 \\
Endurance [min] & 15 & 30 & 30 & 45 & 60 \\
\hline
\end{tabular}

Table 2. MicroHawk performances.

The theoretical results have been compared to the experimental ones, obtained by the wind tunnel testing carried out in the SWT facility at Politecnico di Torino, on a $300 \mathrm{~mm}$ wingspan scaled model of MicroHawk [5]. The experimental tests have been performed by using a 5-components internal balance and provided lateral force and normal force, as well as the aerodynamic moments (Fig. 3).

In order to perform the initial sizing of the propulsive system for the micro aerial vehicle, according to the main mission specifications related to the project, an analytical model for DC motor - propeller system has been developed. Four basic parameters are needed for motor modeling: stall torque, stall current, no load angular velocity and no load current. The propeller performances are estimated by using the blade element approach (2D blade profile aerodynamics is provided). An iterative procedure for the estimation of inflow factors was also implemented. The model has been validated with experimental data for motors and propellers tested at Politecnico di Torino. A 2components external balance has been used to provide thrust and torque on the tested electric engines [5]. Energy storage is a widely acknowledged challenging phase of the micro aerial vehicles design, particularly when total weight constraints strongly limit the energy source weight, although flight performance requirements, such as flight duration, have to be satisfied. As to the MicroHawk design, referring to off-the-shelf components, Li-polymer batteries have been chosen. An analytical model for in-flight battery discharge was adopted.

Flight performances [5] of MicroHawk prototypes were computed by means of a software code in order to obtain the relationship between operational speed and 
required power and thrust. In-flight thrust and power requirements were estimated by conventional performance analysis, after vehicle drag polar estimation. Minimum flight speed, cruise speed and climb rates (depending upon wing loading) were also estimated. An analytical model for propulsion-battery combination was used to evaluate flight range and endurance.

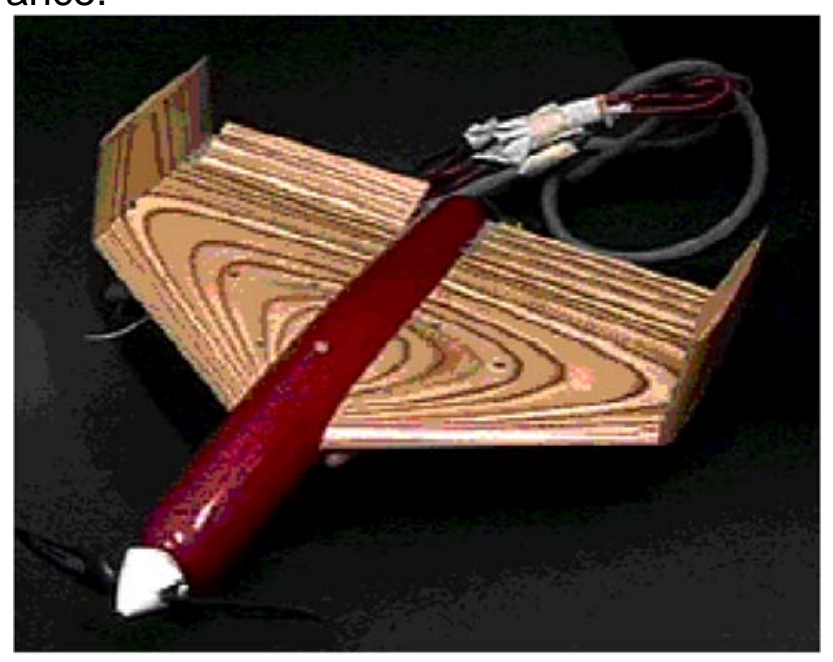

Fig. 3. Experimental model for wind tunnel testing.

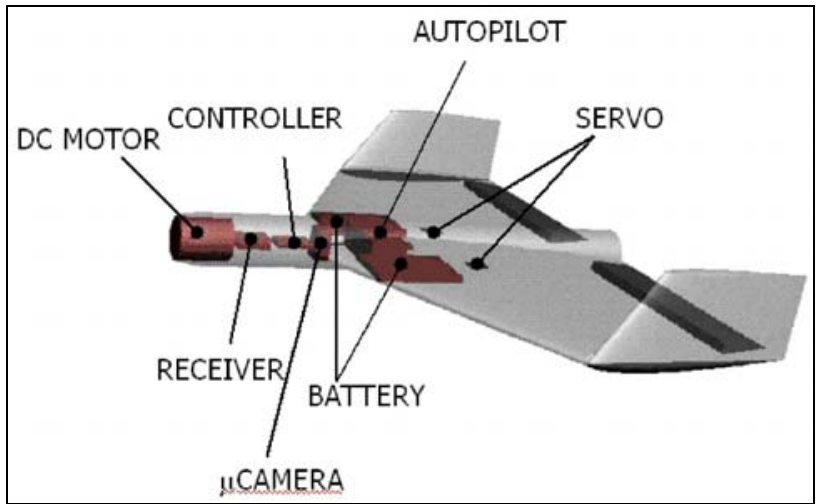

Fig. 4. MicroHawk on-board layout and subsystems (MH600 version).

From the analysis of available references on MAV design, it can be observed that, as a general rule, this last generation of aerial vehicles is designed and developed in terms of expected performances with a trial and error process, which is usually based on reiteration of flight experiments. Several aspects support this empirical approach, which is widely adopted by aeromodelers in the development of radio-controlled airplanes. The design experience on these kinds of vehicles is still limited and the definition of the reference aerodynamic database is not generally straightforward. Even if the vehicles are developed with the help of computational solvers or wind tunnel experiments, part of the stability, damping and control derivatives can be only estimated by means of criteria based on past experiences on similar configurations.

Flight dynamics analysis and preliminary control system design are discouraged by the 
inaccurate prediction of system dynamic response, affected by many model uncertainties (aerodynamics, propulsion system, actuator dynamics, ...). Differently from the large-scale aircraft case, platform stabilization and loop closures (when available) are tuned with flight experiments during the development of the flying prototype.

As an additional remark, the development of flight simulation support for MAV design and remote pilot training is not so effective in terms of advantages for several specific reasons: low cost of prototypes, minimal environmental impact and limited flight test range requirements for line-of-sight remote piloting. Hence, not much of the resources scheduled for the project development are devoted for the assessment and the validation of either analytical or numerical accurate mathematical models of the vehicle. As a consequence, the number of available references in the area of flight dynamics for micro aerial vehicles is quite limited.

A mathematical model of MicroHawk was developed for off-line dynamics analysis and real-time flight simulation [5][7]. It is a nonlinear representation of single engine aircraft with rigid fuselage. No small angle assumption is invoked for aerodynamic angles of the vehicle and the aerodynamics of fuselage and stabilizers is modeled using static coefficients and stability derivatives obtained by wind tunnel experiments at different angles of attack and sideslip angles. The effects of aerodynamic controls (elevator and aileron) are superimposed in terms of increments. The rigid body motion of the aircraft is modeled using six nonlinear force and moment equations and three kinematic relations (Euler equations). The most important feature of these equations of motion is that the states need not to be small quantities; thus, all the kinematic nonlinearities associated with the motion of the rigid body are retained. The propulsive system model based on DC motor and propeller was implemented, including the mathematical correlation between supplying voltage and current drain with engine operating rpm and the relationships between propeller rpm and thrust and torque coefficients.

The flying and handling qualities requirements were considered within the design procedure of MicroHawk platform [8], in order to test the compliance to conventional standards and to relate the dynamics analysis results to the qualitative ratings obtained by flight testing activity. As a result of the open loop dynamics analysis, the need for flying qualities assessment was made evident and it was the basis for a further research development, aimed to the design of a flight control system addressed to the stable closure of the aircraft dynamics loop. Firstly, in an attempt to provide a solution for aircraft controllability, a lag compensator was introduced and it demonstrated the feasibility of a system dynamics improvement. Therefore, a more detailed flight control system design activity was started; as to the gain synthesis methodology, eigenstructure assignment and linear quadratic regulator techniques were evaluated in order to point out the most effective method for aircraft control. A roll-yaw damper was also designed in order to correct the degradation of lateral dynamic stability observed during experiments in pre-stall flight conditions. Finally, a technique for the inclusion of handling qualities requirements in the flight control system design process was tested [8]. The search for an optimal controller in terms of handling qualities was performed numerically with a genetic algorithm. 
A wide activity of real-time flight simulation was carried out to support and validate the flight dynamics theoretical analysis. The real-time dynamics analysis was based on Hexagon $^{\mathrm{TM}}$, a flight simulation software tool addressed to the interaction of a userdefined aircraft mathematical model, a virtual instruments panel and a real-time data analysis graphic interface, flying the aircraft within a virtual scenario. A graphical interface, dedicated to the configuration of the simulation session, allowed to set the aircraft mathematical model (based on linear or non-linear formulation), the starting flight conditions (in terms of flight speed, operating altitude, climb angle, steady turn rate) and the aircraft initial position with respect to an inertial reference frame defined by the scenario designers. The Hexagon ${ }^{\mathrm{TM}}$ system also provided a virtual basic instrumentation panel. The real-time data analysis was used to evaluate the aircraft response to maneuver, the stall behavior, the stability characteristics and the control surfaces effectiveness.

Another concern for the designer is the test of components (receiver, antennas, servo systems, batteries, motors, propellers, cables, ...) and their on-board layout. Subsystems integration was experimentally tested for weight balance and electromagnetic interference by means of virtual and physical mock-ups (see Fig. 4).

Several flight tests were carried out in order to evaluate stability and control characteristics of the MicroHawk configuration. The tested configuration shows to be also controllable during the release from a carrier, stable and controllable in gliding flight, controllable in turns. It is characterized by level flight during landing, low sink rate, capability to withstand wind and to perform minimal aerobatic flight (looping). Performances were also assessed after repeated flight analysis in order to validate the analytical models adopted in the design process.

As the flight-testing experiences demonstrated, the MicroHawk is able to perform whether autonomous or under remote piloting control.

The typical mission profile can be described by the following phases (see Figs. 6 and 7):

- Take-off procedure can consist of hand launch or traditional take off for MH2000. The micro can be also released from a greater sized UAV, acting as a carrier, in order to reach the mission area and the defined operating altitude;

- Whether autonomous or under remote piloting controls are enabled; the former solution should be the only one applicable in the case of out-of-sight flight, due to long mission ranges, higher operating altitude, inaccessible spots or lack of visibility;

- In-flight operations. The platform can take on-board a payload consisting of sensors or microcameras, depending on the mission profile, the geometric sizes and the required power;

- Landing procedure, both for autonomous and remote piloting control, is conventional-like, without ad-hoc systems for assisted landing (i.e. parachute); this solution allows to recover the payload together with the platform itself, without compromising payload physical integrity thanks to the controllable landing phase. 

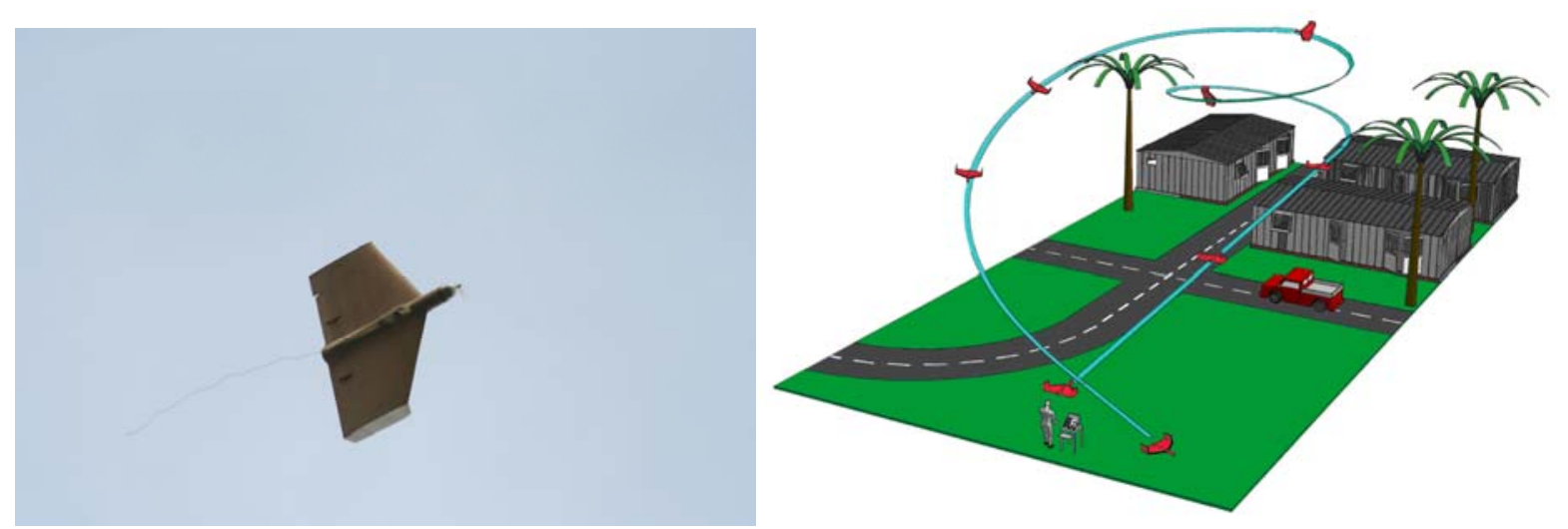

Fig. 5 - MicroHawk in flight experiments (MH600 version).

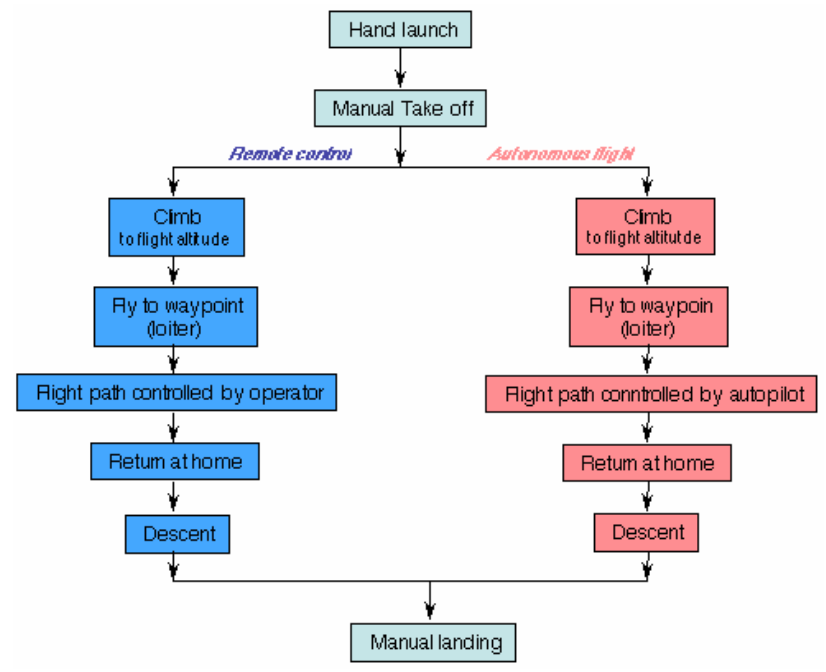

Fig. 6. MicroHawk mission sequence.

Flight test activity is also mandatory to explore the feasibility of practical applications in the real world. As a matter of fact, European civil aviation authorities have recently outlined [9] the limits for in-flight operations of light UAV systems. These restrictions must be clearly kept in mind when the platform should operate in urban areas and/or in presence of operators who could be potentially injured in case of accidental impact. The regulatory restrictions and their application to in-flight operations is a vital aspect. Their practical implications could preclude future development of MAVs. National aviation authorities have an evident role in this field.

\section{PRODUCT DEVELOPMENT}

An industrial version of the MicroHawk platform was derived by MAVTech s.r.l., a spinoff company of Politecnico di Torino. The potential of the company is the production of technical solutions with competitive performances and costs (including customer support and training of end users) deriving from the development of knowledge-based projects. The primary interest is the development of innovative aeronautical solutions for aerial 
surveillance and tactical operation support for civil applications. The design and the production of mini-RPVs and mini-UAVs, the creation of man-portable solutions, the integration with customer applications, the development of specific control and simulation tools are the core activities of MAVTech s.r.I..

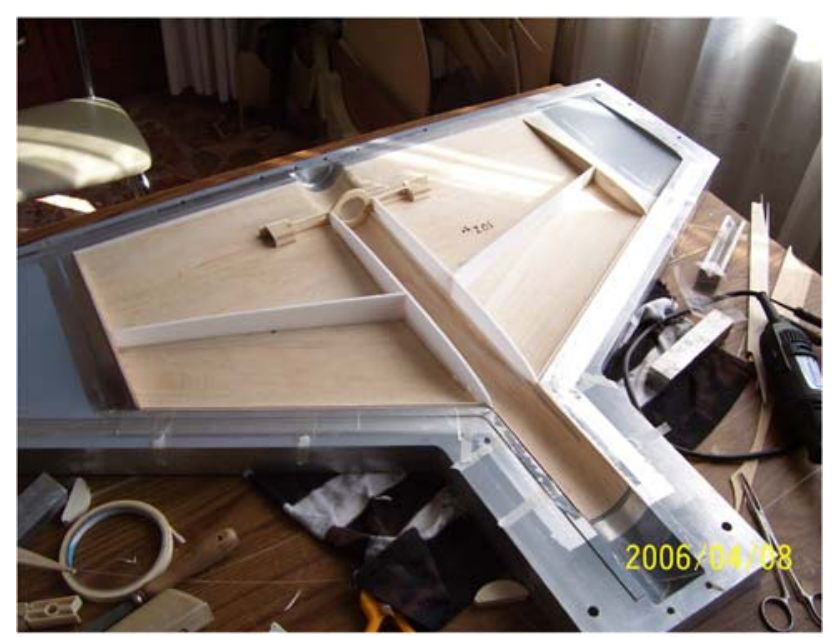

Fig. 7. Production of the airframe (MH600 version).

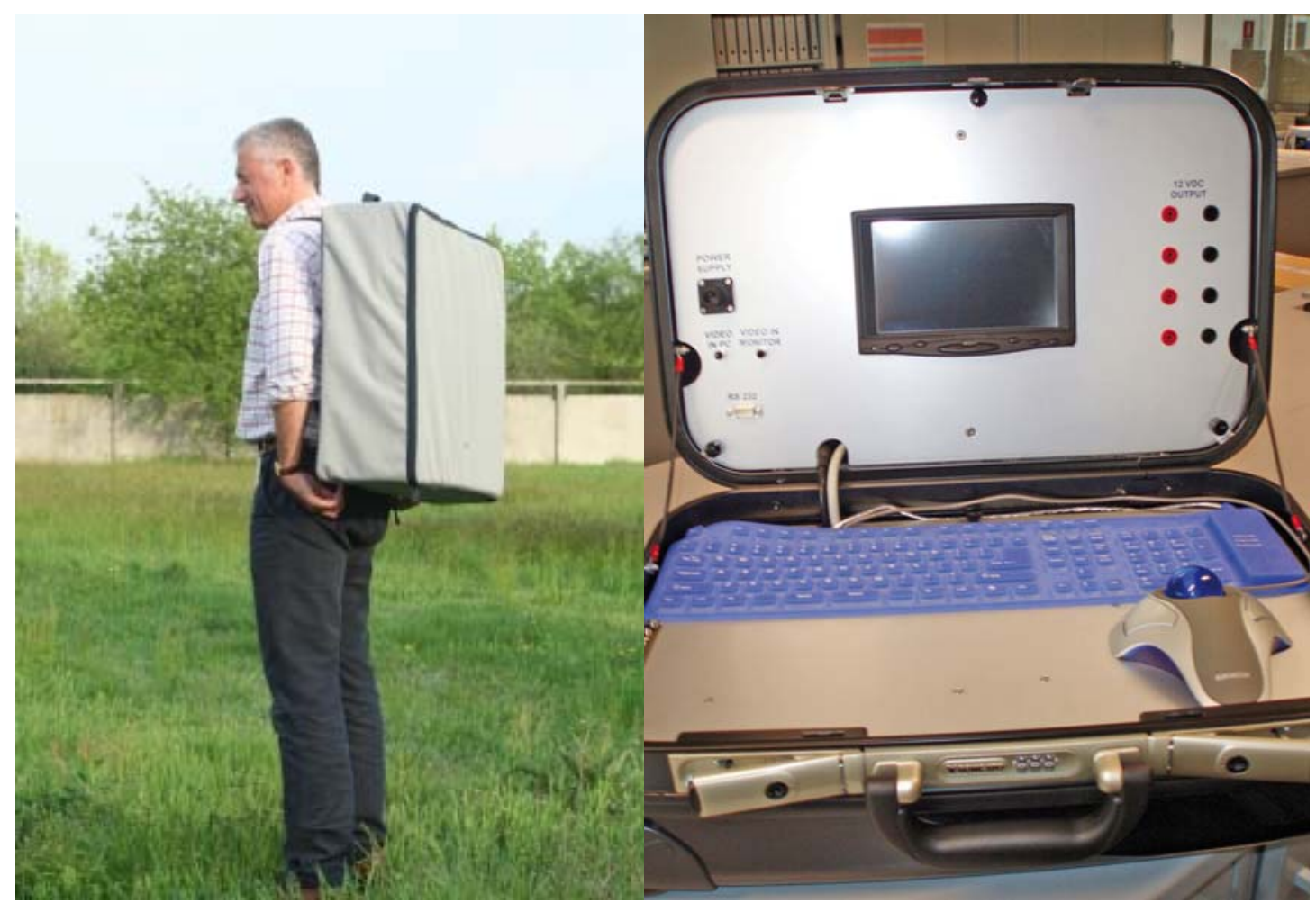

Fig. 8. Man-portable setup and ground control station (MH600 version).

The basic mini-UAV commercial version is based on the MH600 prototype even if the production of larger vehicles is provided on demand. The structure of the airframe is produced after glass fiber mold forming (Fig. 7). This technique ensures shape 
accuracy, reproducibility of airframe weight and geometry, good stability and adequate crash-worthiness. The platform is protected by damage during transport in a manportable backpack (Fig. 8) and the accessories for flight (RC control, battery charger, spare batteries and cables) are also fit into the same fabric bag (Fig. 9).

On-board components are located on a support (sleeve mount in the fuselage) and printed circuits replace wires. A ventral pod was added to the configuration to increase the payload capacity of the platform. Custom payload installations are possible within weight and power limitations. The computer used to program the autopilot is fit within the portable ground control station (Fig. 8). Different serial connectors are available for the data link. A multi-function display is used for terminal output and real time video streaming from the platform. Images and video signals are digitized and stored in a backup mass storage device. The video receiver and eventually the radio modem can be placed within the computer case. Auxiliary voltage supply is also provided for battery charger and other accessories.

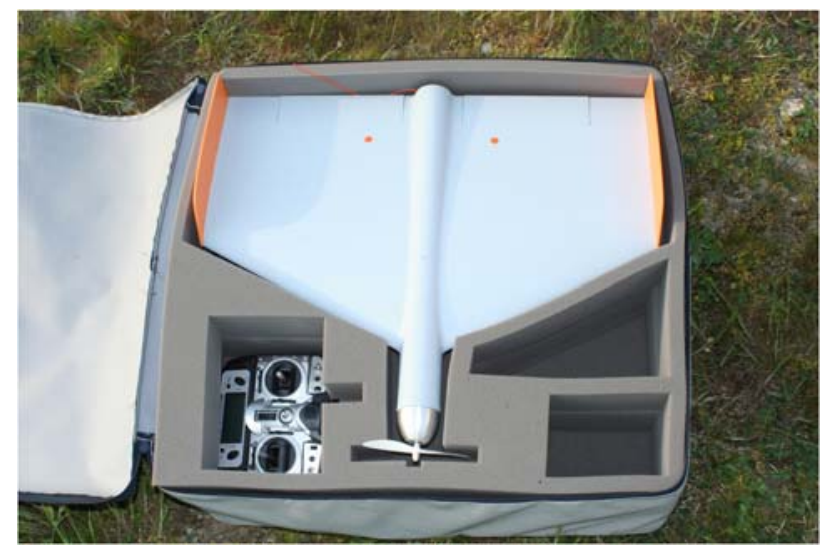

Fig. 9. Man-portable setup (MH600 version).

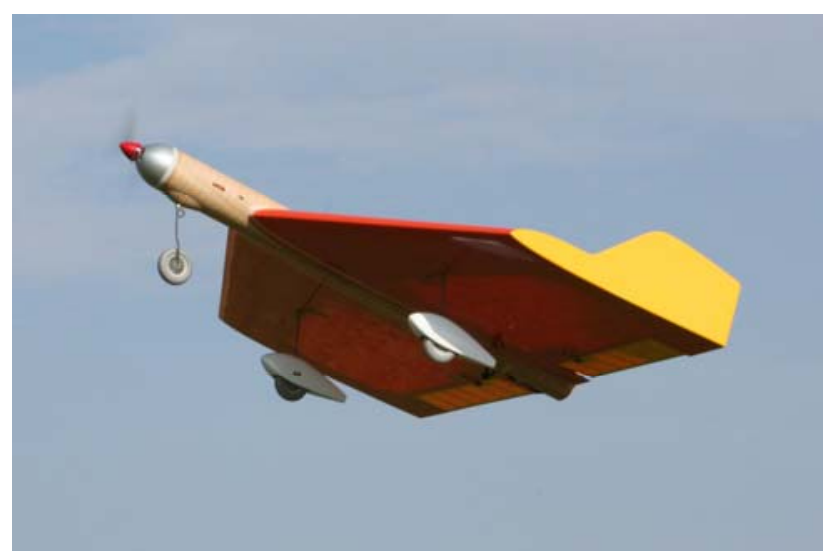

Fig. 10 - MicroHawk in flight experiments (MH2000 version).

An example of larger size platform is presented in Fig. 10. The MH2000 version is produced on demand for larger endurance/payload requirements. A custom ventral pod can be used as removable payload bay for additional sensors and video systems. The components are produced with carbon fiber mold forming. This aircraft is operated with 
conventional take-off and landing (landing gear). A stabilized video camera provides real time line of sight vision that is broadcasted to a ground receiver for surveillance and remote control. A radio link also provides the tracking of the vehicle and the flight parameters including coordinates are continuously reproduced on a digital display (ground control station). The same link is used for remote setup of the autopilot and wireless reconfiguration of mission tasks (update of waypoints).

\section{CONCLUDING REMARKS}

The MicroHawk program was presented. The origin and the evolution of the research program, the theoretical work, the design phases, the experimental tests and, finally, the development of an industrial version of the platform were detailed. As a general comment, the MicroHawk program is a singular example of multi-disciplinary research and development activity with a potential for application over a wide range of useful missions.

\section{REFERENCES}

[1] "Future Technology Driven Revolutions In Military Operations", DARPA, USA, (1992).

[2] UAVNET Thematic Network, Document library, http://www.uavnet.com, (2007).

[3] NASA UAV Applications Center, USA, http://www.uav-applications.org/, (2007).

[4] Lorefice L., Pralio B., Quagliotti F., "Fluorescent Oil Flow Visualization Technique Applied To 2D Airfoils At Very Low Reynolds Numbers", 11th International Symposium On Flow Visualization, Notre Dame, USA, (2004).

[5] Pralio B., Guglieri, G., Quagliotti, F., "Design and Performance Analysis of a Micro Aerial Vehicle Concept", 2nd AIAA Unmanned Unlimited Systems, Technologies and Operations Conference, San Diego, USA, (2003).

[6] "Aircraft Configuration Interface - Software Manual", Rel. 1.0, MAVTech s.r.I., (2006).

[7] Lorefice L., Pralio B., Guglieri G., Quagliotti F., "Tools for Flight Dynamics Analysis in the Design of MAVs", 24th ICAS Congress. Yokohama, Japan, (2005).

[8] Guglieri G., Pralio B., Quagliotti F., "Flight Control System Design for a Micro Aerial Vehicle", Aircraft Engineering and Aerospace Technology, vol. 78, pp. 87-97, (2006).

[9] "UAV Task-Force Final Report”, ANNEX 1, JAA/Eurocontrol, (2004). 